\title{
Artificial Intelligence Segmentation Model-Based Computed Tomography Angiography Image in the Diagnosis of Congenital Aortic Constriction
}

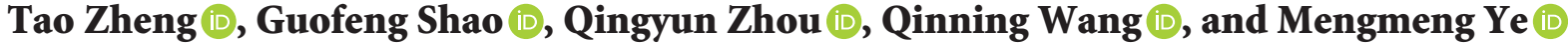 \\ Department of Cardiothoracic and Vascular Surgery, Ningbo Medical Center Lihuili Hospital, Ningbo 315000, Zhejiang, China \\ Correspondence should be addressed to Guofeng Shao; 1651010038@xzyz.edu.cn
}

Received 6 October 2021; Revised 11 December 2021; Accepted 14 December 2021; Published 7 January 2022

Academic Editor: M Pallikonda Rajasekaran

Copyright (c) 2022 Tao Zheng et al. This is an open access article distributed under the Creative Commons Attribution License, which permits unrestricted use, distribution, and reproduction in any medium, provided the original work is properly cited.

\begin{abstract}
This study was to analyze the impacts of the image segmentation model and computed tomography angiography (CTA) on the clinical diagnosis of aortic constriction under the background of artificial intelligence. In this study, 126 patients with congenital aortic constriction (CAC) diagnosed by surgery were selected as the research objects and routine digital subtraction angiography (DSA) and CTA were performed. Then, the traditional active contour model (AC model) was optimized based on the local area information to construct a new image segmentation model for intelligent segmentation and reconstruction of the CTA images of patients. The results revealed that compared with the AC model and the image segmentation model based on region growth (RG model) obtained from angiography segmentation, the algorithm constructed in this study showed a smaller segmentation range for angiography images and more accurate segmentation results. The quantitative data results suggested that the evolution times and running time of the constructed model were less than those of the AC and RG models $(P<0.05)$. Based on the gold standard of DSA examination results, there were 122 correctly diagnosed cases, 3 missed diagnosed cases, and 1 misdiagnosed by CTA, so the diagnosis coincidence rate was $96.83 \%$. Compared with DSA, the average inner diameter and average pressure difference of patients with precatheter, paracatheter, and postcatheter type were not greatly different in CTA $(P>0.05)$. The CTA examination suggested there were 154 cases with intracardiac structural abnormalities, with a detection rate of $86.52 \%$; there were 32 cases of cardiac-vascular connection abnormalities, with a detection rate of $100 \%$; and there were 79 extracardiac vascular abnormalities, with the detection rate of $95.18 \%$. It indicated that the optimized image segmentation model based on local area information proposed in this paper has excellent segmentation performance for CT angiography images and has good segmentation effect and efficiency. The CTA based on the artificial intelligence image segmentation model showed a better diagnostic effect on abnormal heart-vascular connection and abnormal extracardiac blood vessels and can be used as an effective examination method for clinical diagnosis of CAC.
\end{abstract}

\section{Introduction}

Congenital aortic constriction (CAC) is a congenital heart disease characterized by narrowing of the ductus arteriosus or near the aortic ligament. It can occur alone, or it can be combined with the Turner syndrome, two-leaflet aortic valve, atrial septal defect, ventricular septal defect, and other congenital heart diseases [1]. Depending on the location and degree of stenosis, the symptoms of CAC may manifest as hypertension, lower extremity arterial ischemia, heart failure, intracranial aneurysm rupture, etc. $[2,3]$. The cause of aortic constriction is not yet fully understood. There are two main theories: one is that during fetal development, the muscular tissue in the arterial duct extends into the aortic wall and then shrinks and fibrosis leads to narrowing of the corresponding part of the aortic lumen; the other is that during the fetal period, the blood flow pattern of the fetal heart is different from that of adults due to the presence of the arterial catheter, the right ventricle pumps the blood through the arterial catheter to supply the lower body, and the left ventricle pumps blood directly to the upper limbs and head, causing it to flow through the aortic isthmus so 
that the blood flow is reduced, leading to dysplasia of the aorta in the corresponding part [4-7]. Therefore, when children or adults have severe headaches, nosebleeds, and intermittent breaks, it is necessary to go to the hospital for diagnosis in time.

Early and accurate diagnosis is very important for the treatment and prognosis of aortic constriction. At present, the clinical diagnosis of aortic constriction often uses chest $\mathrm{X}$-ray, computed tomography (CT), magnetic resonance imaging (MRI), ultrasound, contrast, and other imaging methods [8]. Among them, chest radiographs are cheap and suitable for preliminary screening of aortic constriction, but their diagnostic sensitivity is too low. The color Doppler ultrasound can accurately assess the degree of aortic stenosis and the corresponding heart and valve abnormalities. CT images can perform three-dimensional (3D) reconstruction of diseased blood vessels and clearly identify the location of the lesion and its surrounding conditions, but there is ionizing radiation, which is not suitable for repeated examinations [9]. Computed tomography angiography (CTA) is a non-invasive method to assess the pressure gradient of aortic constriction, which can accurately assess the collateral circulation. When the patient undergoes interventional treatment, CTA can be used to solve the problem at one time. In this study, CTA was intended applied to diagnose and evaluate patients with CAC.

With the rapid development of artificial intelligence and computer technology, medical intelligent diagnosis has become an auxiliary means of clinical imaging examination. Image segmentation refers to the division of regions according to the color and gray of the image, and then the use of computer models to merge and adjust the key features, and finally achieve the purpose of target recognition. In the field of medicine, intelligent segmentation can improve the quality of the original image to a certain extent, and assist physicians in providing the reliability of diagnosis results $[10,11]$. Therefore, it is very important to choose an appropriate image segmentation model. In this study, 126 patients with CAC diagnosed by surgery were selected as the research objects and underwent the routine digital subtraction angiography (DSA) and CTA examinations. Then, the traditional active contour model (AC model) was optimized based on the local area information to construct a new image segmentation model for intelligent segmentation and reconstruction of the CTA images of patients. The DSA examination result was undertaken as the gold standard to evaluate the diagnostic performance of the CTA images based on the image segmentation model for CAC.

\section{Materials and Methods}

2.1. Research Samples. 126 patients with CAC diagnosed by surgery who were admitted to the hospital from May 1, 2017, to May 1, 2020, were selected as the research subjects, and all were examined by conventional DSA and CTA. Among them, there were 81 males and 45 females, ranging in age from 15 to 43 years old, with an average age of $26.72 \pm 7.95$ years old. This study had been approved by the ethics committee of the hospital, and the patients and their families had understood the situation of the study and signed the informed consent forms.

The inclusion criteria were defined as follows: patients with clinical symptoms such as heart murmur, dyspnea, chest tightness, and fatigue; patients with good treatment compliance; patients under 15 years old; patients with complete clinical data. The exclusion criteria were given as follows: patients with aortic constriction by pure echocardiogram; patients who gave up treatment and had been discharged; patients who had not been confirmed by surgery; patients who were older than 70 years old.

2.2. CTA Examination. 64 rows 128-slice Lightspeed spiral CT was adopted to examine all patients. The specific steps were as follows. The patient was required to keep in a supine position, breathe calmly, and stop swallowing activities, with the shoulders drooping naturally. The head was fixed with a head frame so that the orbital line was perpendicular to the bed surface. The scan was from the entrance of the patient's thorax to the upper mid-abdomen position. After a plain scan, a $320 \mathrm{mg} / \mathrm{mL}$ non-ionic contrast agent iopamidol (injection speed of $2-5 \mathrm{~mL} / \mathrm{s}$ ) was injected from the elbow vein, and relevant data were collected at the same time. The scanning parameters were set as follows: the voltage was $100 \mathrm{kV}$, the current was $120 \mathrm{~mA}$, the matrix was $256 \times 256$, and the scanning time was 5-7 seconds.

2.3. DSA Examination. The patient was required to keep in a supine position. After local anesthesia with $3 \%$ lidocaine, the vascular puncture was applied at the most obvious right femoral artery pulsation (the needle angle was about $35^{\circ}$ ). Then, the $5 \mathrm{~F}$ arterial sheath was inserted and fixed and rinsed with heparin saline. Under the guidance of the loach guidewire, a $5 \mathrm{~F}$ Cordis pigtail radiography tube was used for contrast (dose of $26 \mathrm{~mL}$, and a flow rate of $15 \mathrm{~mL} / \mathrm{s}$ ). Next, $4 \mathrm{~F}$ Cordis singlecurved catheter was adopted for cerebral angiography (dose of $12 \mathrm{~mL}$, and a flow rate of $8 \mathrm{~mL} / \mathrm{s}$ ), which can clearly display the common carotid artery, internal and external carotid arteries, vertebra-basal artery, middle cerebral artery, anterior cerebral artery, posterior cerebral artery, and external blood vessels. The conventional frontal, lateral, and oblique images were taken to dynamically observe the condition of blood vessels. The contrast agent was iohexol injection onipex (concentration at $150 \mathrm{mg} / \mathrm{mL}$ ). During the operation, we had to pay attention to the detection of the patient's vital signs such as breathing, heart rate, and blood pressure. Immediately after the operation, manual compression was adopted to stop bleeding for about 18 minutes until there was no bleeding at the puncture point when there was no compression. After that, an elastic bandage was applied to compress for 24 hours. The patient was instructed to puncture the lower limbs and brake 24 hours, or a professional suture device or vascular occluder can be adopted to stop the bleeding.

2.4. Image Processing and Evaluation. The collected original images were transmitted to the workstation and diagnosed by two imaging physicians with more than 5 years of work 
experience. The aortic arch and the three major branch vessels of the patient were observed from multiple angles to check whether there were collateral arteries and analyze the anatomical structure and adjacent relationship of the heart and great vessels. In addition, the pathological anatomy of the patient's aortic constriction was recorded, and the diameter and length of the constriction were measured.

Image quality evaluation can be graded into three levels: clear (the vascular shadow was clear and sharp and the image was complete without artifacts and bone occlusion), good (the blood vessel wall was smooth, but there was a small amount of skull base or cervical skeletal artifacts), and poor (the vascular shadow was blurred, and there were more multiple bone artifacts).
2.5. Optimized Image Segmentation Model Based on Local Area Information. The traditional AC (active contour) [12] can detect holes in the image, is insensitive to the position of the initial contour curve and has nothing to do with the image gradient information and can better adapt to images with large and small gradient changes. However, because this model only uses global information, it cannot correctly segment images with the uneven grayscale distribution. Therefore, the local area information was introduced in this study to optimize the traditional AC model. An original image was supposed to be $P$, then its energy function can be expressed as below equation:

$$
F\left(L, l_{1} \cdot l_{2}\right)=\alpha_{1} \int_{\text {inside }}\left|P(x)-l_{1}(x)\right|^{2} \mathrm{~d} x+\alpha_{2} \int_{\text {outside }}\left|P(x)-l_{2}(x)\right|^{2} \mathrm{~d} x+\beta H(L)
$$

In equation (1), $\alpha_{1}$ was the internal energy weight parameter, $\alpha_{2}$ was the external energy weight parameter, $L$ represented the contour curve, $\beta$ referred to the weight parameter of the perimeter of the contour curve, $x$ was any point in the image, and $F()$ was the energy function. In addition, different from the traditional active contour $\left(l_{1}(x)\right.$ and $l_{2}(x)$ were the mean gray values inside and outside the contour curve, respectively), both $l_{1}(x)$ and $l_{2}(x)$ were functions, and their values were related to the image information of the local area around the $x$ point. Figure 1 is a schematic diagram of $l_{1}(x)$ and $l_{2}(x)$ of the AC model before and after optimization, where $a$ was any point inside the contour curve $L$, and $b$ was any point outside the contour curve $L$.

During the optimization of the AC model, $l_{1}(x)$ and $l_{2}(x)$ could be expressed as follows:

$$
\begin{aligned}
& l_{1}(x)=\frac{\left[P(x) G_{v}(\phi(x))\right]^{\diamond} K_{R}(x)}{G_{v}(\phi(x))^{\diamond} K_{R}(x)}, \\
& l_{2}(x)=\frac{\left[P(x) 1-G_{v}(\phi(x))\right] * K_{R}(x)}{\left(1-G_{v}(\phi(x))\right) * K_{R}(x)} .
\end{aligned}
$$

In the abovementioned two equations, $\diamond$ referred to the convolution symbol, $G()$ represented the Heaviside function, $\phi()$ was the level set function, $K_{R}$ ( ) was the kernel function $\left(K_{R}(x)=\left(\operatorname{sign}\left(R^{2}-x^{2}\right)+1\right) / 2\right)$, and $R$ was the radius of the kernel function. This method enabled the average gray value of any $x$ point only related to the gray values of other points near the $x$ point and changed the uniform constant corresponding to all points of the image into a function of each point corresponding to the local area of each point, thereby solving the problem that the traditional AC model can only use global information and cannot apply to the problem of uneven brightness images. Then, the Heaviside function [13] and Dirac function [14] were introduced to transform this function into a level set function, then the below equation can be obtained:

$$
F\left(\phi, l_{1}, l_{2}\right)=\eta_{1} \int_{\Omega}\left|P(x)-l_{1}(x)\right|^{2} G(\phi) \mathrm{d} x+\eta_{2} \int_{\Omega}\left|P(x)-l_{2}(x)\right|^{2}(1-G(\phi)) \mathrm{d} x+\beta H(\phi)
$$

The level set function would be distorted in actual operation and cannot meet the condition of meeting the signed distance function, so it was necessary to constantly initialize the level set function, which greatly increased the workload of the operation, so a new solution had to be introduced in actual calculations.

$$
\begin{aligned}
\phi(x) & =\operatorname{sign}\left(\phi_{0}\right)(1-|\Delta \phi|) . \\
\phi(x, y, 0) & =\phi_{0} .
\end{aligned}
$$

Equations (4) and (5) were the signed distance functions, and $|\Delta \phi|=1$. In order to further realize the automatic 


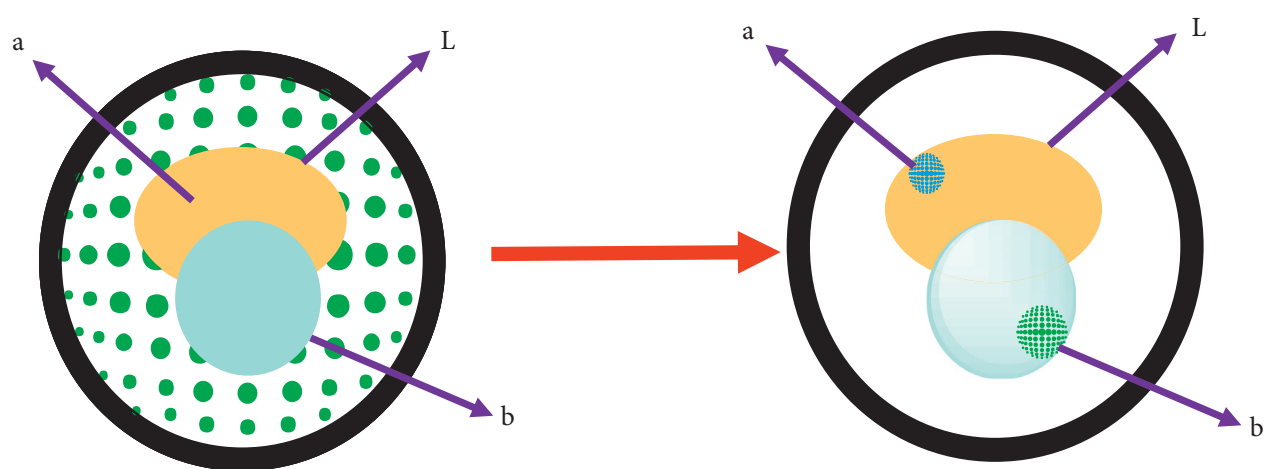

FIGURE 1: Schematic diagram of $l_{1}(x)$ and $l_{2}(x)$ of the AC model before and after optimization. The left figure shows the schematic diagram of $l_{1}(x)$ and $l_{2}(x)$ of the traditional AC model, and the right figure shows the schematic diagram of $l_{1}(x)$ and $l_{2}(x)$ of the optimized AC model.

orthodontics of the function, the energy constraint was introduced.

$$
E *=\int_{\Omega} \frac{|\Delta \phi(x)-1|^{2}}{2} \mathrm{~d} x .
$$

The below equation can be obtained by calculating equation (6) using the gradient descent method [15]:

$$
\frac{\partial \phi}{\partial t}=\operatorname{div}\left(1-\frac{\Delta \phi}{|\Delta \phi|}\right) .
$$

In equation (7), $1-(1 /|\Delta \phi|)$ was the diffusion factor. If $|\Delta \phi|>1$, then $\phi$ would be smoother; if $|\Delta \phi|<1$, then $\phi$ would be steeper. The equation (8) could be obtained by incorporating equation (7) into the level set function.

$$
F *\left(\phi, l_{1}, l_{2}\right)=F\left(\phi, l_{1}, l_{2}\right)+\lambda E *\left(\phi, l_{1}, l_{2}\right)
$$

$\lambda$ was the weight parameter of the orthodontic function in the abovementioned equation. In actual calculations, the following regularization functions were also introduced [16]:

$$
\begin{aligned}
G_{u}(x) & =\frac{(2 \arctan / \pi)(x / u)+1}{2}, \\
\zeta_{u}(x) & =\frac{u}{\pi\left(u^{2}+x^{2}\right)} .
\end{aligned}
$$

The gradient flow that minimized the energy function can be obtained by differentiating the equation (8):

$$
\begin{aligned}
& \frac{\partial \phi}{\partial t}=\frac{\partial F}{\partial \phi} \\
& \frac{\partial \phi}{\partial t}=-\delta_{u}(\phi)\left[\eta_{1}\left(F-l_{1}\right)^{2}-\eta_{2}\left(F-l_{2}\right)^{2}\right]+\beta \delta_{u}(\phi) \Gamma+\lambda(\Delta \phi-\Gamma) .
\end{aligned}
$$

In the abovementioned equations, $-\delta_{u}(\phi) \eta_{1}\left(F-l_{1}\right)^{2}$ represented the regional energy, $\beta \delta_{u}(\phi) \Gamma$ represented the length term function, $\lambda(\Delta \phi-\Gamma)$ referred to the orthodontic function, $\Gamma$ was the curvature of the level set and
$\Gamma(x)=(\operatorname{div} \Delta \phi /|\Delta \phi|)$, and $\Delta$ represented the Laplacian operator and $\Delta(\phi)=\nabla(\nabla(\phi))$. Thus, the final function can be expressed as follows:

$$
\phi_{k+1}=\phi_{k}+\Delta t\left\{-\delta_{u}(\phi)\left[\eta_{1}\left(P-l_{1}\right)^{2}-\eta_{2}\left(P-l_{2}\right)^{2}\right]+\beta \delta_{u}(\phi) \Gamma+\lambda(\diamond \phi-\Gamma)\right\} .
$$

In the above equation (11), $\Delta t$ was the evolution time step of the level set. The basic flow of the model is shown in Figure 2.

2.6. Image Segmentation Simulation Experiment. The experimental platform was Matlab7.0 software, the central processing unit (CPU) is $2.5 \mathrm{GHz}$, and the memory was 3 GB. The image segmentation model optimized in this study was adopted for the initial segmentation experiment on the simple artificially synthesized noisy image. The evolution times of segmentation should be recorded. Then, the optimized image segmentation model, traditional AC model, and RG (region growth) model [17] were applied to the angiography images to verify the effectiveness of segmentation. The evolution times and running time required for the model segmentation were selected as indicators to quantitatively evaluate the performance of the model. 


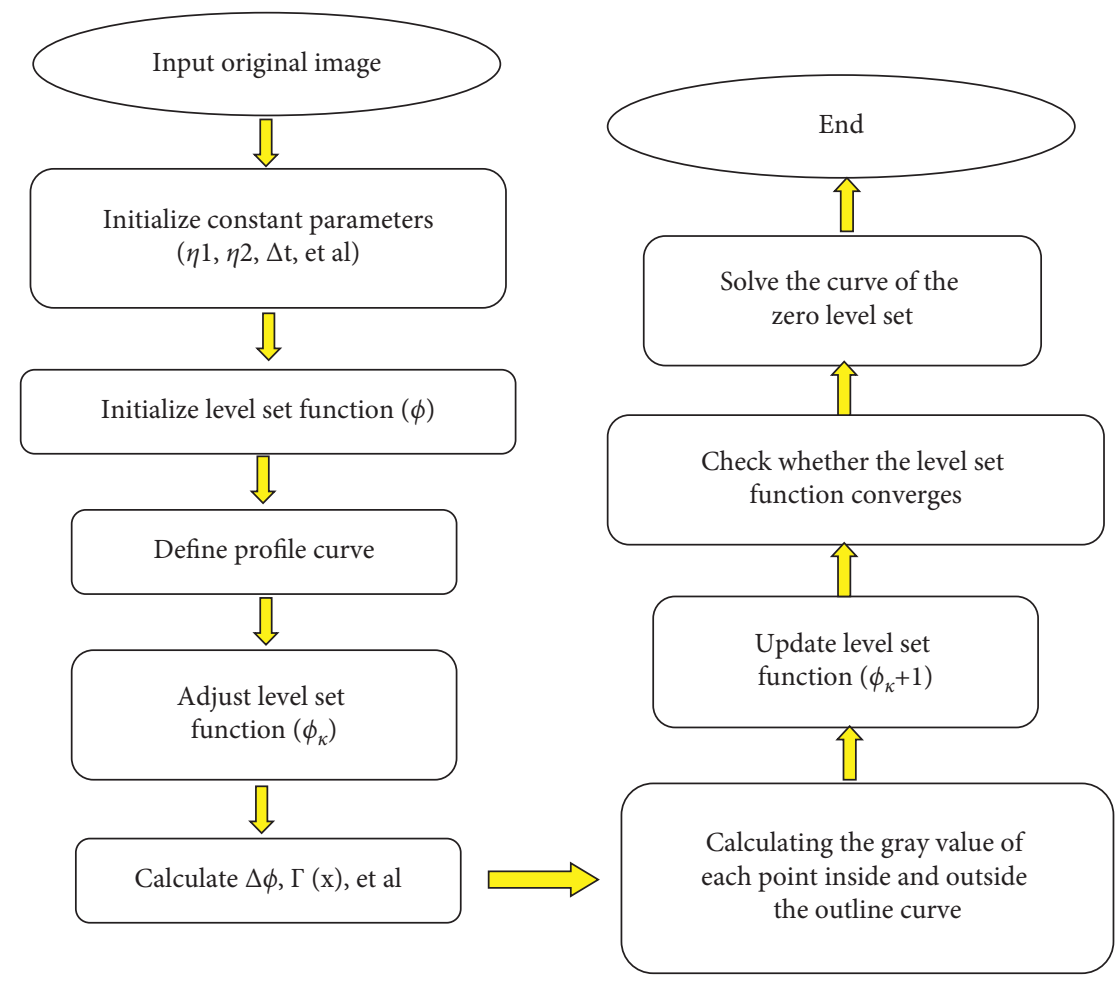

FIgURE 2: Optimized image segmentation models based on local area information.

2.7. Statistical Methods. The data processing of this study was analyzed by SPSS 19.0 version statistical software, the measurement data was expressed by the mean \pm standard deviation $(\bar{x} \pm s)$, and the count data was expressed by the percentage (\%). The one-way analysis of variance was used for pairwise comparison. The difference was statistically significant at $P<0.05$.

\section{Results}

3.1. Image Segmentation Experiment Results. Figure 3 shows the segmentation effect of the artificially synthesized noise map by the optimized image segmentation model. The position of the contour curve was constantly changing under different evolution steps, and the evolution was only related to the local area of the point. When the evolution was performed for 95 steps, the segmentation effect of the optimized model was the best.

Figure 4 shows the segmentation effect of three models on CTA. It can be seen that compared with AC and RG models, the model constructed in this study showed a smaller segmentation range for angiography images, and the segmentation results were more accurate, which can display all important information.

In order to further clarify the image segmentation performance of the model constructed in this paper, quantitative index comparisons were performed. Figure 5 illustrates the evolution times and running time required for the image segmentation of the three models. Figures 5(a) and 5(b) reveal that the evolution times and running time required by the construction model were much less than those of the AC and RG models, and the differences were statistically significant $(P<0.05)$.

3.2. Analysis of Constriction Types of Patients. As shown in Figure 6, the DSA examination confirmed 68 cases of precatheter, 32 cases of paracatheter, and 26 cases of postcatheter. If the DSA examination result was undertaken as the gold standard, there were 122 correctly diagnosed cases, 3 missed diagnosed cases, and 1 misdiagnosed by CTA, so the diagnosis coincidence rate was $96.83 \%$.

Figure 7 illustrates the results of DSA and CTA examinations for different types of constriction. The average inner diameter and average pressure difference of precatheter, paracatheter, and postcatheter patients in the CTA examination were not statistically significant compared with those of the DSA $(P>0.05)$.

Figure 8 shows a CTA image of a male patient (42 years old). The clinical symptoms include that precordial squeezing pain occurred when tired, lasting for 2 hours, which can be relieved after rest; the image showed the local uterus of the descending aortic arch, and the cavity was constricted. Therefore, it was diagnosed as aortic constriction. Figure 9 shows a CTA image of a female patient (35 years old). The clinical symptoms included emotional agitation after a full meal, or chest tightness and shortness of breath caused by fatigue, which could be relieved after rest. The physical signs were audible and continuous murmurs in the second intercostal space on the left edge of the sternum. The image showed patent ductus arteriosus, and there were circulating blood vessels between the aortic arch and the pulmonary artery. Therefore, it 


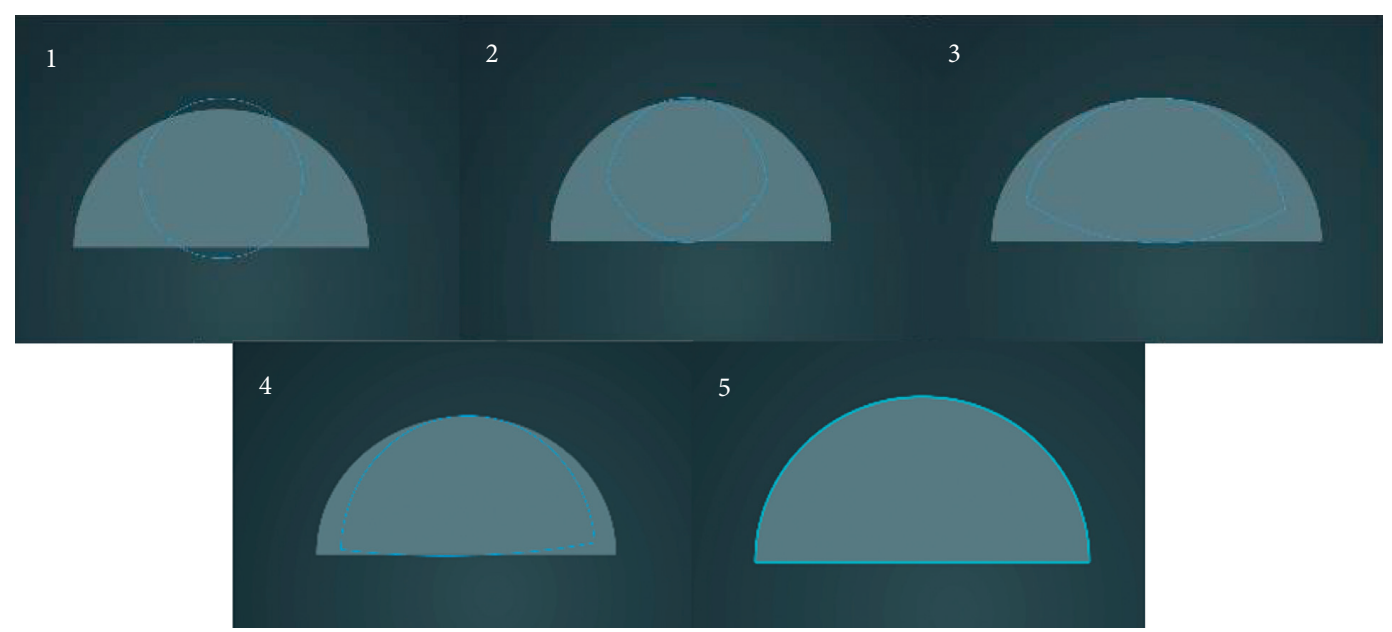

FIGURE 3: The segmentation effect of the optimized image segmentation model on the artificially synthesized noise map. 1-5 were the original image and contour lines, those after 15 steps of evolution, those after 45 steps of evolution, those after 65 steps of evolution, and those after 95 steps of evolution.

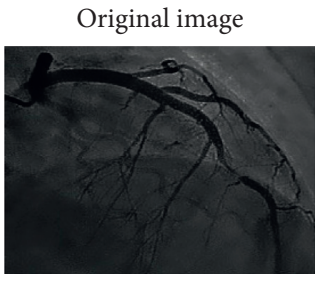

Original image
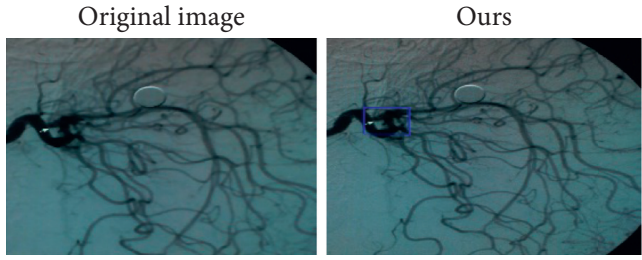

AC

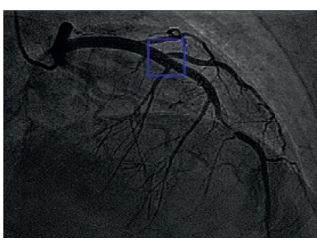

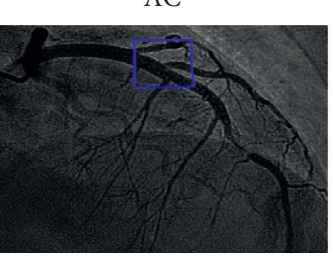

(a)

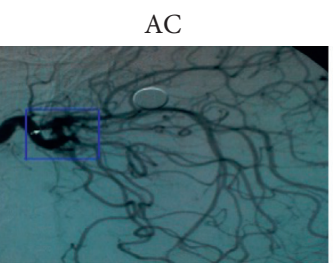

(b)
RG

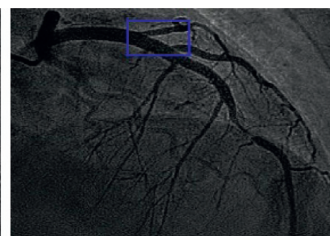

RG

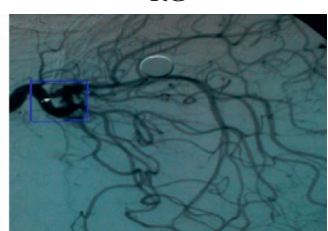

Figure 4: The segmentation effect of three models on CTA. (a, b) CT angiography images.

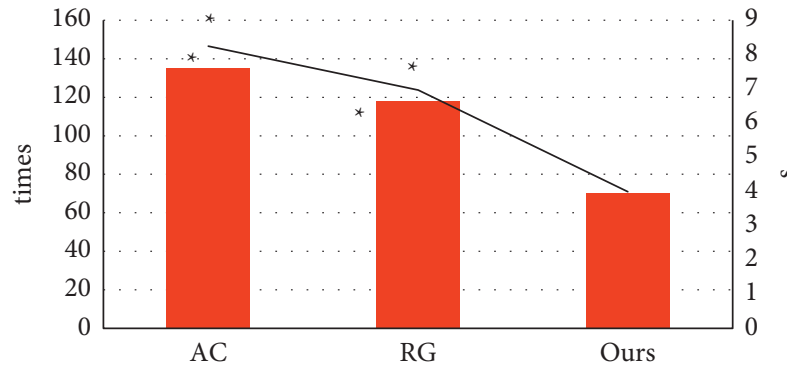

Evolution times — Time

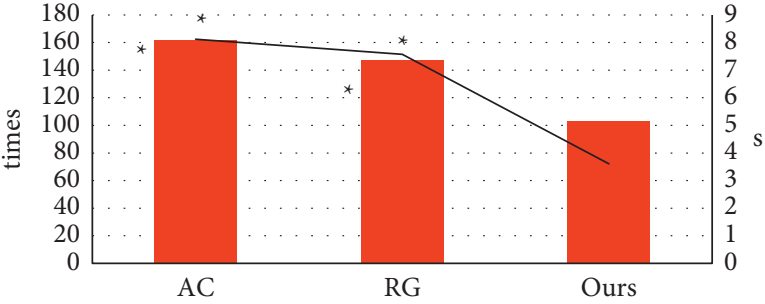

Evolution times — Time

(a)

(b)

FiguRE 5: Evolution times and running time required for image segmentation of the three models. (a) The segmentation index; (b) the segmentation index. * indicates that the difference was statistically obvious in contrast to the constructed model $(P<0.05)$. 


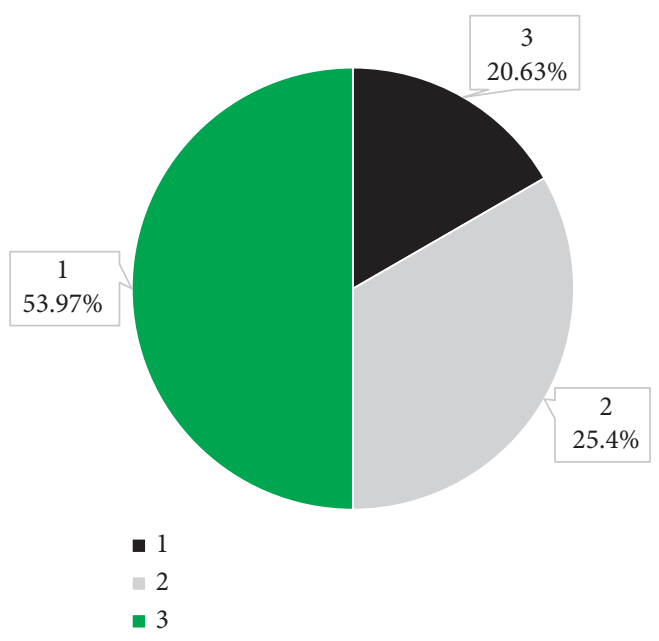

FiguRE 6: DSA examination results of different constriction types. 1, 2, and 3 referred to precatheter, paracatheter, and postcatheter, respectively.

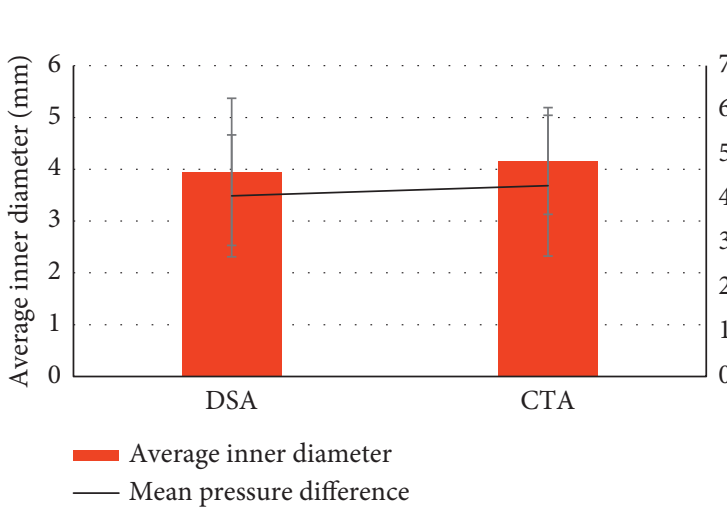

(a)

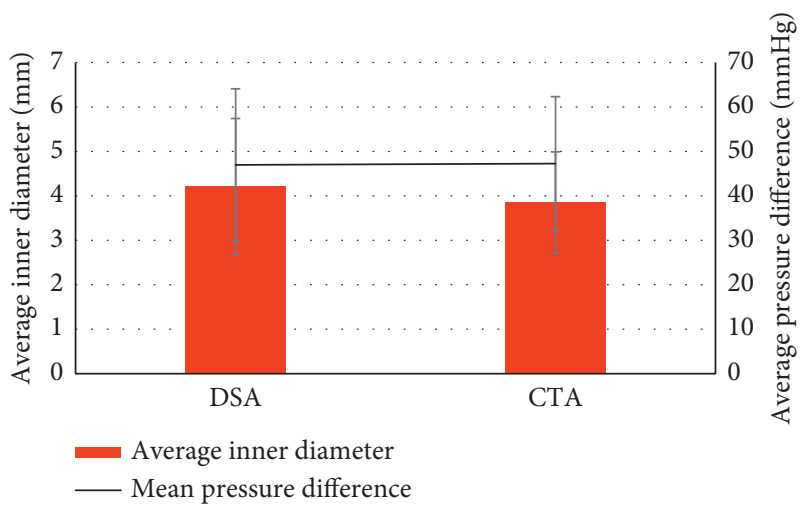

(b)

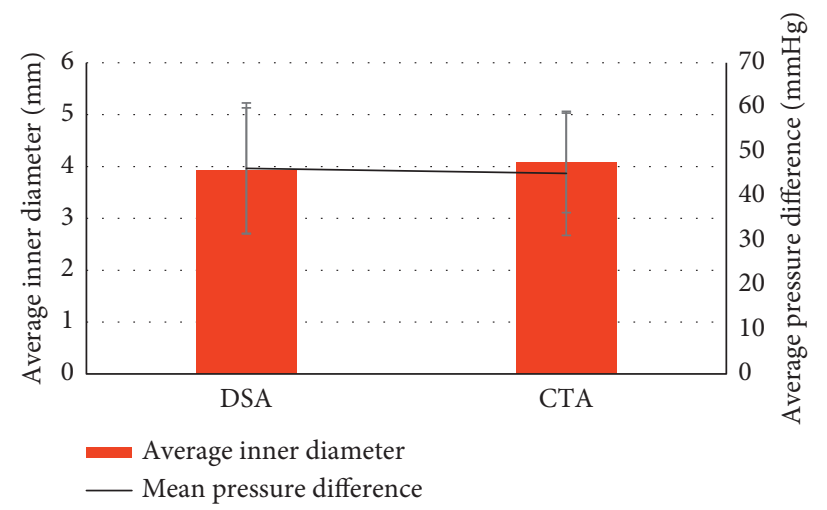

(c)

FIGURE 7: DSA and CTA examination results of different constriction types. (a-c) The results of precatheter, paracatheter, and postcatheter types, respectively.

was diagnosed as aortic constriction with patent ductus arteriosus.

3.3. Combined Malformations of Patients. Figure 10 shows the results of the DSA examination of the patients with abnormalities. There were 293 cases of combined abnormalities, including 178 cases of intracardiac structural abnormalities, 32 cases of cardiac-vascular connection abnormalities, and 83 cases of extracardiac vascular abnormalities. Figure 11 shows the results of the CTA examination. If the DSA result was undertaken as the gold standard, the CTA examination suggested that there were 154 cases with intracardiac structural abnormalities, with a 


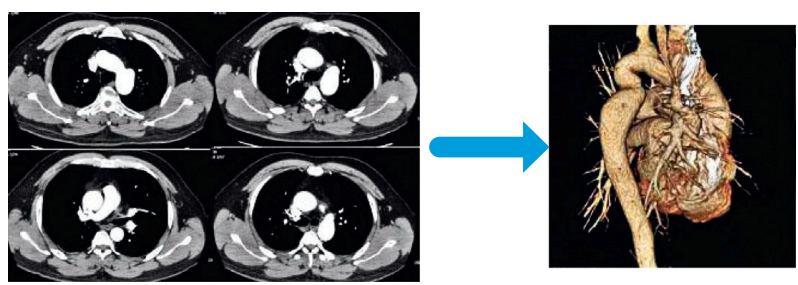

Figure 8: CTA images of a male patient. The left image was the CTA image and the right image was the 3D reconstruction image using the aortic volume reconstruction technology.

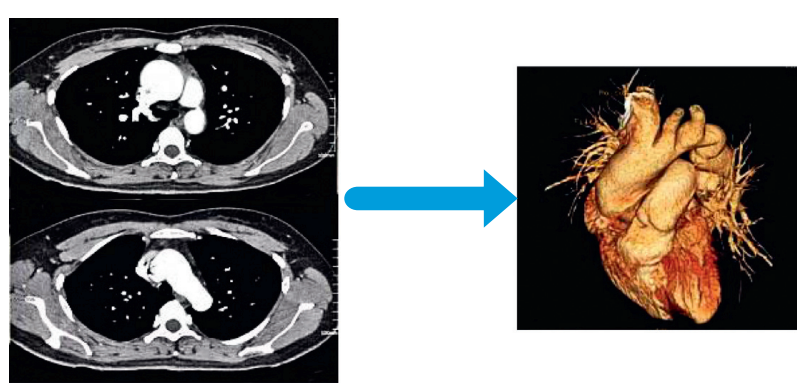

FIgURE 9: CTA images of a female patient. The left image was the CTA image and the right image was the 3D reconstruction image using the aortic volume reconstruction technology.

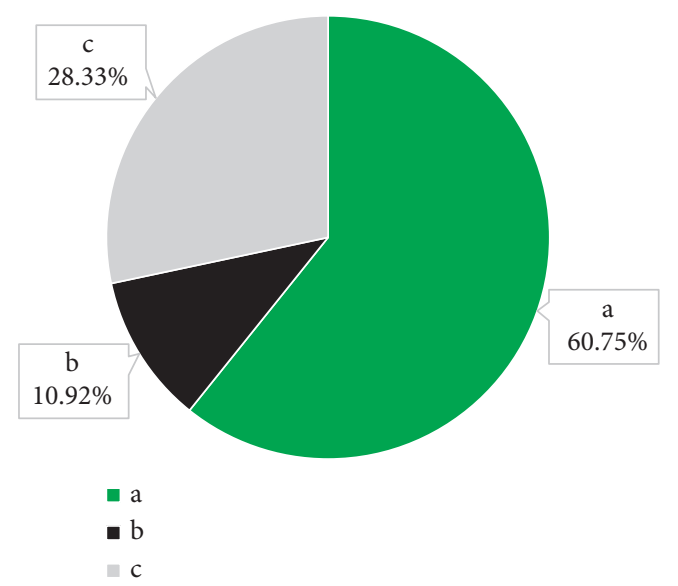

FIgURE 10: DSA examination results of patients with malformations. $(a-c)$ The percentages of patients with intracardiac structural abnormalities, cardiac-vascular connection abnormalities, and extracardiac vascular abnormalities, respectively.

detection rate of $86.52 \%$; there were 32 cases of cardiacvascular connection abnormalities, with a detection rate of $100 \%$; and there were 79 extracardiac vascular abnormalities, with the detection rate of $95.18 \%$. In addition, 4 cases were missed, including 2 cases of patent ductus arteriosus, 1 case of aortic septal constriction, and 1 case of abnormal brachiocephalic veins.

\section{Discussion}

Aortic constriction refers to the congenital stenosis of the local lumen of the aorta, which is generally concentrated near the junction of the arterial duct and the aortic duct.
According to the positional relationship between the stenosis and the arterial duct, it can be divided into a precatheter constriction, paracatheter constriction, and postcatheter constriction $[18,19]$. There are many clinical examination methods for aortic constriction, including echocardiography, CTA, DSA, and MRI. In order to further improve the diagnostic effect of aortic constriction, the traditional AC model was optimized in this study based on local area information to construct a new image segmentation model, which was applied to 126 cases of CAC patients diagnosed by surgery in the CTA angiography diagnosis. For the segmentation effect of the simple artificially synthesized noise map, it can be seen that the position of the contour curve was constantly changing under different evolution steps, and the evolution was only related to the local area of the point. When the evolution was 95 steps, the segmentation effect of the model optimized in this study achieved the best performance. Compared with the AC and RG models, the model constructed showed a smaller segmentation range for angiography and more accurate segmentation result, which can display all important information. The quantitative data results suggested that the evolution times and running time of the constructed model were less than those of the AC and RG models $(P<0.05)$, which was similar to the results of Wang et al. [20], indicating that the image segmentation performance of this model was better.

The DSA examination confirmed 68 cases of precatheter, 32 cases of paracatheter, and 26 cases of postcatheter. If the DSA examination result was undertaken as the gold standard, there were 122 correctly diagnosed cases, 3 missed diagnosed cases, and 1 misdiagnosed by the CTA, so the diagnosis coincidence rate was $96.83 \%$. Such results indicated that CTA imaging shows high accuracy for the 


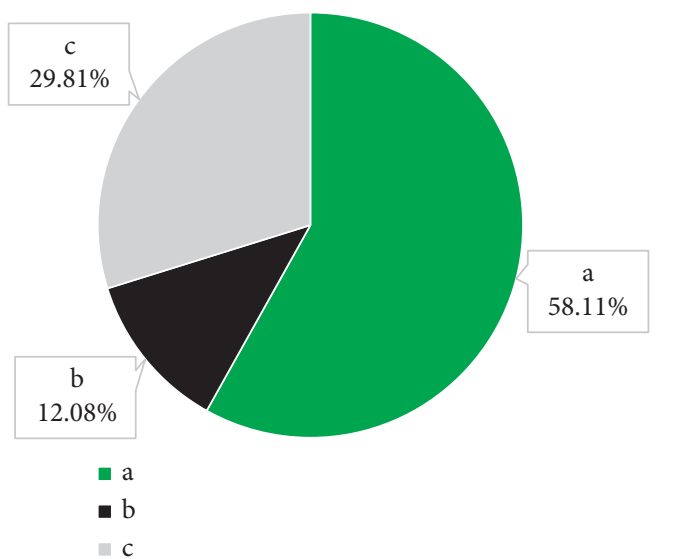

(a)

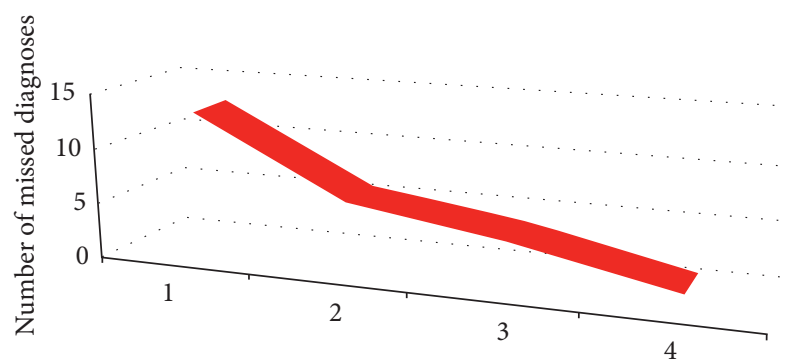

(b)

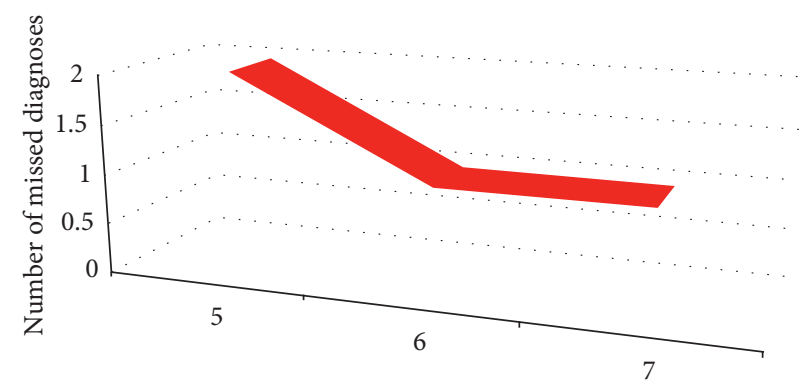

(c)

FIGURE 11: CTA examination results of patients with malformations. (a-c) The percentages of patients with intracardiac structural abnormalities, cardiac-vascular connection abnormalities, and extracardiac vascular abnormalities, respectively. 1-7 represent the valvular disease, patent foramen ovale, atrial septal defect, endocardial cushion defect, patent ductus arteriosus, aortic diaphragm type constriction, and abnormal brachiocephalic vein, respectively.

diagnosis of aortic constriction. The individual missed diagnosis and misdiagnosis may be related to the differences in clinical experience and picture quality of physicians themselves [21]. Compared with DSA, the average inner diameter and average pressure difference of patients with precatheter, paracatheter, and postcatheter type were not greatly different in CTA $(P>0.05)$, suggesting that the CTA imaging based on the new image segmentation model showed the equivalent accuracy to that of the DSA, and it was also suitable for clinical diagnosis. The results of the DSA examination showed that there were 293 cases of combined abnormalities, including 178 cases of intracardiac structural abnormalities, 32 cases of cardiac-vascular connection abnormalities, and 83 cases of extracardiac vascular abnormalities. The DSA is a common method for clinical observation of vascular diseases, and its diagnostic accuracy is extremely high $[22,23]$. If the DSA result was undertaken as the gold standard, the CTA examination suggested there were 154 cases with intracardiac structural abnormalities, with a detection rate of $86.52 \%$; there were 32 cases of cardiac-vascular connection abnormalities, with a detection rate of $100 \%$; and there were 79 extracardiac vascular abnormalities, with the detection rate of $95.18 \%$. It revealed that the CTA based on the new image segmentation model showed a better diagnostic effect on the cardiovascular- vascular connection abnormalities and extracardiac vascular abnormalities [24, 25].

\section{Conclusion}

In this study, the traditional AC model was optimized based on the local area information to construct a new image segmentation model, which was applied to the CTA diagnosis of 126 CAC patients diagnosed by surgery. The results found that the CTA imaging based on the artificial intelligence image segmentation model showed a better diagnostic effect on cardiovascular-vascular connection abnormalities and extracardiac vascular abnormalities and can be used as an effective means of clinical diagnosis of CAC. The optimized image segmentation model based on local area information proposed has excellent segmentation performance for CT angiography images and has good segmentation effect and efficiency. However, there were some shortcomings in this study. The number of aortic constriction patient samples was little with a single source, which may affect the feasibility of the results. In the followup, it will increase the selection of patient samples and further analyze the new image segmentation model constructed, so as to improve the reliability of the model. In a word, the research of combining artificial intelligence 
technology with imaging in this paper provides a reference for the intelligent evaluation of congenital coarctation of the aorta.

\section{Data Availability}

The data used to support the findings of this study are available from the corresponding author upon request.

\section{Conflicts of Interest}

The authors declare that there are no conflicts of interest.

\section{References}

[1] Y. Yu, Y. Wang, M. Yang et al., "Evaluating the severity of aortic coarctation in infants using anatomic features measured on CTA," European Radiology, vol. 31, no. 3, pp. 1216-1226, 2021.

[2] C. Guo, J. Lu, Z. Tian, W. Guo, and A. Darvishan, "Optimization of critical parameters of PEM fuel cell using TLBODE based on Elman neural network," Energy Conversion and Management, vol. 183, pp. 149-158, 2019.

[3] T. Szmuda, P. Słoniewski, S. Ali, A. Kamieniecki, and J. Dzierżanowski, "Extent of anterior clinoidectomy for clipping of carotid-ophthalmic aneurysms predicted on threedimensional computerised tomography angiography," Neurologia I Neurochirurgia Polska, vol. 54, no. 2, pp. 138-149, 2020.

[4] M. Hu, Y. Zhong, S. Xie, H. Lv, and Z. Lv, "Fuzzy system based medical image processing for brain disease prediction," Frontiers in Neuroscience, vol. 15, Article ID 714318, 2021.

[5] B. Tamarappoo, Y. Otaki, M. Doris et al., "Improvement in LDL is associated with decrease in non-calcified plaque volume on coronary CTA as measured by automated quantitative software," Journal of Cardiovascular Computed Tomography, vol. 12, no. 5, pp. 385-390, 2018.

[6] H. Zhang and L. Feng, "Coarctation of the aorta complicated with intracranial aneurysm: a case report and literature review," World Neurosurgery, vol. 112, pp. 25-30, 2018.

[7] P. Wang and R. Jiang, "320-slice computed tomography angiography imaging findings and follow-up in A patient with aortic coarctation misdiagnosed as hypertension: a case report and literature review," Cureus, vol. 11, no. 12, Article ID e6529, 2019.

[8] K. Spanos, P. Nana, G. Kouvelos et al., "Factors associated with elimination of type II endoleak during the first year after endovascular aneurysm repair," Journal of Vascular Surgery, vol. 71, no. 1, pp. 56-63, 2020.

[9] S. J. Al'Aref, A. Su, H. Gransar et al., "A cross-sectional survey of coronary plaque composition in individuals on non-statin lipid lowering drug therapies and undergoing coronary computed tomography angiography," Journal of Cardiovascular Computed Tomography, vol. 13, no. 2, pp. 99-104, 2019.

[10] Y. Y. Dai, Z. X. Huang, X. T. Liu, and Q. Z. Wang, "[Risk factors for recurrence of large atherosclerotic cerebral infarction]," Nan Fang Yi Ke Da Xue Bao, vol. 37, no. 12, pp. 1678-1682, 2017, Chinese.

[11] J. A. Ladapo, J. M. Pfeifer, J. M. Pitcavage, B. A. Williams, and A. A. Choy-Shan, "Quantifying sex differences in cardiovascular care among patients evaluated for suspected ischemic heart disease," Journal of Women's Health, vol. 28, no. 5, pp. 698-704, 2019.
[12] S. Shin, H.-B. Park, H.-J. Chang et al., "Impact of intensive LDL cholesterol lowering on coronary artery atherosclerosis progression," Journal of the American College of Cardiology: Cardiovascular Imaging, vol. 10, no. 4, pp. 437-446, 2017.

[13] K. Nakao, T. Noguchi, Y. Asaumi et al., "Effect of eicosapentaenoic acid/docosahexaenoic acid on coronary high-intensity plaques detected with non-contrast T1-weighted imaging (the AQUAMARINE EPA/DHA study): study protocol for a randomized controlled trial," Trials, vol. 19, no. 1, p. 12, 2018.

[14] S. Trefely, C. D. Lovell, N. W. Snyder, and K. E. Wellen, "Compartmentalised acyl-CoA metabolism and roles in chromatin regulation," Molecular Metabolism, vol. 38, Article ID 100941, 2020.

[15] L. Milke and J. Marienhagen, "Engineering intracellular malonyl-CoA availability in microbial hosts and its impact on polyketide and fatty acid synthesis," Applied Microbiology and Biotechnology, vol. 104, no. 14, pp. 6057-6065, 2020.

[16] V. Tillander, S. E. H. Alexson, and D. E. Cohen, "Deactivating fatty acids: acyl-CoA thioesterase-mediated control of lipid metabolism," Trends in Endocrinology and Metabolism, vol. 28, no. 7, pp. 473-484, 2017.

[17] C. E. Bowman and M. J. Wolfgang, "Role of the malonyl-CoA synthetase ACSF3 in mitochondrial metabolism," Advances in Biological Regulation, vol. 71, pp. 34-40, 2019.

[18] M. J. Levy, D. C. Montgomery, M. E. Sardiu et al., "A systems chemoproteomic analysis of acyl-CoA/protein interaction networks," Cell Chemical Biology, vol. 27, no. 3, pp. 322-333, 202019.

[19] A. Ronowska, A. Szutowicz, H. Bielarczyk et al., "The regulatory effects of acetyl-CoA distribution in the healthy and diseased brain," Frontiers in Cellular Neuroscience, vol. 12, p. 169, 2018.

[20] T. Wang, W. Yao, J. Li, Q. He, Y. Shao, and F. Huang, "AcetylCoA from inflammation-induced fatty acids oxidation promotes hepatic malate-aspartate shuttle activity and glycolysis," American Journal of Physiology - Endocrinology And Metabolism, vol. 315, no. 4, pp. E496-E510, 2018.

[21] Z. Wan, Y. Dong, Z. Yu, H. Lv, and Z. Lv, "Semi-supervised support vector machine for digital twins based brain image fusion," Frontiers in Neuroscience, vol. 15, Article ID 705323, 2021.

[22] R. Callari, D. Fischer, H. Heider, and N. Weber, "Biosynthesis of angelyl-CoA in Saccharomyces cerevisiae," Microbial Cell Factories, vol. 17, no. 1, p. 72, 2018.

[23] S. Xie, Z. Yu, and Z. Lv, "Multi-disease prediction based on deep learning: a survey," Computer Modeling in Engineering and Sciences, vol. 127, no. 3, pp. 1-34, 2021.

[24] J. T. Ku, A. Y. Chen, and E. I. Lan, "Metabolic engineering design strategies for increasing acetyl-CoA flux," Metabolites, vol. 10, no. 4, p. 166, 2020.

[25] S. Li, Q. Zhang, J. Wang, Y. Liu, Y. Zhao, and Y. Deng, "Recent progress in metabolic engineering of Saccharomyces cerevisiae for the production of malonyl-CoA derivatives," Journal of Biotechnology, vol. 325, pp. 83-90, 2021. 